\section{Sensibilizándonos para disminuir las discapacidades del lenguaje en niños con antecedentes de prematuridad extrema}

\section{Sr. Editor:}

En relación al artículo "Dificultades del lenguaje en niños preescolares con antecedente de prematuridad extrema" de Mariangela Maggiolo L. publicado en su Revista, concordamos que los recién nacidos prematuros en general y los de prematuridad extrema en particular constituyen un grupo poblacional de alto riesgo susceptible de presentar secuelas en diferentes áreas de su desarrollo, entre ellas dificultades del lenguaje.

Las dificultades de lenguaje y habla son las discapacidades más prevalentes en la población infantil; tienen impacto negativo en el desarrollo global del niño, tanto en sus relaciones sociales como en su vida afectiva, en el logro de habilidades cognitivas $\mathrm{y}$, posteriormente, en el aprendizaje escolar ${ }^{1}$.

La identificación de los niños que presentan retraso del lenguaje dentro del período preescolar debería de ser una prioridad para el pediatra. Es recomendable remitir al logopeda ante toda sospecha de retraso/trastorno del lenguaje. La etapa infantil ( 3 a 5 años) es básica en la intervención, ya que al final de ésta las bases del lenguaje ya están prácticamente adquiridas. Toda detección no llevada a cabo en la etapa preescolar puede afectar negativamente a la adquisición del lenguaje y posteriormente presentarse otros trastornos de aprendizaje. Debe derivarse para estudio a Neuropediatría en caso de retraso global, trastorno específico del lenguaje, disartria o afasia adquirida ${ }^{2}$.

Las poblaciones de niños prematuros tienen, a lo largo de su vida, mayores necesidades, no solamente desde el punto de vista sanitario sino también educacional, social y psicológico, necesidades que se amplían a su familia (la intervención sobre los padres puede favorecer la evolución neurocognitiva). Es imprescindible establecer programas de seguimiento coordinados que incluyan ayuda al crecimiento, ayuda escolar, servicios de la comunidad y conexión con el cuidado primario. En definitiva, ayudas de un gran número de profesionales que permitan el diagnóstico precoz de los retrasos del desarrollo y de las discapacidades, y el control y tratamiento adecuados $^{3}$.

En el Hospital Hipólito Unanue de TacnaPerú, en el año 2013 se presentó 4,9\% de recién nacidos prematuros y $15,16 \%$ de ellos eran extremo bajo peso; en el último quinquenio también se ha incrementado la sobrevida y disminuido la morbilidad de este grupo poblacional.

Sugerimos: 1) Incorporar a la guía de práctica clínica de recién nacidos prematuros la evaluación periódica de la comunicación y el lenguaje; 2) Desarrollar un programa de capacitación y sensibilización a los profesores del sector educación para la detección temprana de patología del lenguaje y derivación oportuna al especialista; 3) Implementar un programa de sensibilización y consejería a los padres y/o personas que tengan a cargo niños con antecedentes de prematuridad extrema a fin que interioricen la magnitud y la importancia de la intervención temprana.

Dada las características del sistema de salud del estado peruano, consideramos que mucho de lo que se pueda mejorar en éstos niños dependerá del interés de los padres informados.

Agradecemos a la Méd. Gina Rossi B., jefa del Servicio de Neonatología del Hospital Hipólito Unanue de Tacna, por habernos proporcionado los datos estadísticos.

Lourdes Lombardi B. Médico Pediatra, Hospital Hipólito Unanue-Tacna, Docente Universidad Privada de Tacna. Bartolomé Iglesias $\boldsymbol{S}$. Médico Cirujano Pediatra, Docente Universidad Privada de Tacna. Jubilado Hospital Hipólito Unanue-Tacna.

Orietta Barriga $S$.

Docente Universidad Privada de Tacna. Giovanna Cuneo A. Docente Universidad Privada de Tacna. 


\section{Referencias}

1.- Schonhaut BL, Maggiolo LM: Alteraciones del lenguaje en la población preescolar. Otorrinolaringologia. Suplemento de Salud (i) Ciencia ISSN 1667-8982. Vol.7, $\mathrm{N}^{\circ}$ 04-Julio 2012.

2.- Gassió-Subirachs R: Trastornos del lenguaje. An Pediatr Contin 2006; 4: 140-4. - Vol. 4 Núm.2 DOI: 10.1016/ S1696-2818(06)73602-2.
3.- Rico Vales T, Herencia Solano C, García Martín A, González Castro S, Puyol Buil PJ, Torres Mohedas J: Programa de Seguimiento prematuro desde el punto de vista terapéutico y educativo. Rev Pediatr Aten Primaria vol.12 no.45 Madrid Jan.-Mar. 2010.

\section{Dirección autor responsable:}

Lourdes Lombardi Bacigalupo

Correo electrónico: lourdeslombardi1@hotmail.com 